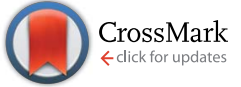

Cite this: Chem. Sci., 2015, 6, 4086

\title{
III-defined concepts in chemistry: rigid force constants vs. compliance constants as bond strength descriptors for the triple bond in diboryne
}

\author{
Jörg Grunenberg*
}

\begin{abstract}
In a recent publication, the interpretation of Braunschweig's diboryne NHC-BB-NHC as a true triple bond is questioned. The analysis by Köppe and Schnöckel is based, inter alia, on the calculation of rigid coupling force constants. Nevertheless, since it is known for a long time that the use of rigid force constants as bond strength descriptors is by no means straightforward, we recomputed the rigid force constants for a model diboryne, applying different coordinate systems and compared the values with the relaxed force constants (generalized compliance constants, GCC). In contrast with the results by Schnöckel and Köppe, the true coupling between the boron-boron bond and the boron-carbon bond, that is, after the elimination of all numerical artifacts, is negligible $\left(f_{\mathrm{BB} / \mathrm{BC}}=-0.003\right)$.
\end{abstract}

Received 13th April 2015

Accepted 2nd May 2015

DOI: $10.1039 / c 5 s c 01322 d$

www.rsc.org/chemicalscience

\section{Computations}

\section{Introduction}

Computational chemistry has reached a high degree of maturity and comprehension making it one of the most active research areas in modern chemical and physical research in general. Predictions concerning single molecules, molecular clusters or even the solid state in combination with detailed information from apparatus based experiments are currently providing the ingredients to an auspicious revolution in the borderland between theory and experiment. Many, but not all, computational chemistry applications deal with observable properties. Here, one can always try to find an experiment, which allows either falsification or confirmation of the computer simulation. This is in sharp contrast to the second major application area of computational chemistry, the underpinning of chemical concepts, where a comparison with experiment is not always possible. Therefore, from time to time, even seemingly trivial questions frequently lead to highly active discussions in the scientific community. Especially the idea of bond orders and localized orbitals, are under debate. ${ }^{1}$

In a recent publication, ${ }^{2}$ the interpretation of the boronboron triple bond in Holger Braunschweig's diboryne NHC-BBNHC $\left(\mathrm{NHC}=\mathrm{N}\right.$-heterocyclic carbene) ${ }^{3}$ as a triple bond is questioned. The analysis by Köppe and Schnöckel is based, inter alia, on the calculation of rigid coupling force constants. Nevertheless, since it is known for a long time that the use of rigid force constants as bond strength descriptors is by no means straightforward, ${ }^{4}$ we conducted the present study in order to evaluate the robustness of the numerical data.

Institut für Anorganische und Analytische Chemie, Technische Universität Braunschweig, Braunschweig 38106, Germany. E-mail: Joerg.Grunenberg@tu-bs.de
To analyze the numerical stability of rigid coupling force constants, as computed by Schnöckel und Köppe in ref. 2, we calculated, in a first step, the $3 \times 3$ matrix for $\mathrm{H}_{2} \mathrm{O}$ at the $\operatorname{CCSD}(\mathrm{T})$ /aug-cc-pvqz level of theory. Scheme 1 shows the matrix of force constants for water in terms of two different coordinate systems (I and II). Both systems have in common the two stretching coordinates $\mathrm{OH}(1)$ and $\mathrm{OH}(2)$. They differ only with respect to the third coordinate, which is the angle $\mathrm{H}-\mathrm{O}-\mathrm{H}$ or the $\mathrm{H} \cdots \mathrm{H}$ "stretching" coordinate, respectively. The rigid force constant matrix (coordinate system I) predicts a $\mathrm{OH}$ bond strength of 8.34 mdyn $\AA^{-1}$, while after transformation into coordinate system II the $\mathrm{OH}$ force constant significantly changes to 8.97 mdyn $\AA^{-1}$, simulating a stronger bond. Even more importantly, the stretch/stretch coupling constant does not only dramatically change its absolute value (from 0.11 to 0.47 mdyn $\AA^{-1}$ ), again simulating a strong electronic coupling, but also the algebraic sign from - to +. Any interpretation as a bond strength descriptor, is thus invalid. On the other side, looking at the relaxed force constant matrices (compliance matrix $^{5}$ ) expressed in both coordinate systems I and II, a numerically stable sub-matrix can be identified. With 8.34 mdyn $\AA^{-1}$, the value of the relaxed force constant (inverse of the compliance constant) depicts a lower threshold of all possible coordinate systems. The same is true for the coupling constants. Both, value and sign, are no longer dependent on the coordinate system. In fact, the real $\mathrm{OH} / \mathrm{OH}$ coupling constant vanishes completely.

Coming back to the diboryne question, the seemingly high $\mathrm{BB} / \mathrm{BC}$ coupling force constant computed by Schnöckel and Köppe of +0.16 mdyn $\AA^{-1}$ piques one's curiosity: is it "real” or is it an artifact of the coordinate system selected by the authors? 


\begin{tabular}{|c|c|c|c|}
\hline $\begin{array}{c}\text { rigid } \\
\text { force constants }\end{array}$ & $Q r$ & ${ }^{2} 2$ & 0,0 \\
\hline & 8.34 & -0.11 & 0.24 \\
\hline & -0.11 & 8.34 & 0.24 \\
\hline & 0.24 & 0.24 & 0.69 \\
\hline
\end{tabular}

coordinate system I

\begin{tabular}{|c|c|c|c|}
\hline $\begin{array}{l}\text { relaxed force } \\
\text { constants }\end{array}$ & & ${ }^{2} \propto$ & \\
\hline & 8.26 & 0 & -0.04 \\
\hline & 0 & 8.26 & -0.04 \\
\hline & -0.04 & -0.04 & 0.65 \\
\hline
\end{tabular}

coordinate system I

\begin{tabular}{|c|c|c|c|}
\hline $\begin{array}{c}\text { rigid } \\
\text { force constants }\end{array}$ & $2 r$ & ${ }^{2} \propto$ & $Q_{13}$ \\
\hline & 8.97 & 0.47 & -1.15 \\
\hline & 0.47 & 8.97 & -1.15 \\
\hline r3 0 & -1.15 & -1.15 & 1.19 \\
\hline
\end{tabular}

coordinate system II

\begin{tabular}{|c|c|c|c|}
\hline $\begin{array}{c}\text { relaxed force } \\
\text { constants }\end{array}$ & $\Theta_{0}^{\mathrm{r} 1}$ & $\mathrm{r}^{\mathrm{r} 2} \mathrm{R}_{\mathrm{r}}$ \\
\hline $\mathrm{R}_{0}^{\mathrm{r} 1}$ & 8.26 & 0 & 0.07 \\
\hline $\mathrm{r} 2 \mathrm{R}_{0}$ & 0 & 8.26 & 0.07 \\
\hline $\mathrm{r}_{0}$ & 0.07 & 0.07 & 1.69 \\
\hline
\end{tabular}

coordinate system II

Scheme 1 The matrices of rigid force constants (upper row) and relaxed force constants (lower row) for water computed at the CCSD(T)/augccpv5z level of theory.

This is important since, in the words of Schnöckel and Köppe "the interaction force constant means the interaction between two bonds, i.e. whether or not and to which extent there is a restoring force within the two bonds".

As part of our ongoing project to develop unique numerical descriptors for chemical concepts, we introduced the method of generalized compliance constants $(G C C)$ some years ago, as an extension to the theory of compliance matrices, valid for arbitrary non-stationary or stationary points on the potential energy hypersurface. ${ }^{6}$ The entries of the inverted Hessian matrix, the compliance constants, do not suffer from coordinate dependencies and can thus be assumed to be much more transferable between similar chemical environments. ${ }^{7}$

In order to separate real coupling phenomena from deceptive ones suggested by numerical artefacts, we - in a second step - therefore recomputed (1) the rigid force constants for Schnöckel's model system $\mathbf{1}$ of Braunschweig's diboryne, applying three different coordinate systems (see below) and (2) relaxed force constants applying our $G C C$ formalism. In the following we denote the different coordinate systems according to Schnöckel's and Köppe's symmetry coordinates (S1); Peter Pulay's natural internal coordinates (S2); primitive internal z-matrix coordinates (S3) and finally our own generalized compliance coordinates, ${ }^{\boldsymbol{6} \boldsymbol{b}}$ consisting of a redundant set of stretching coordinates (S4). For a better comparison with Schnöckel's data, all geometry optimizations and Cartesian force constants were computed at the BP87/dz level of theory. The transformation of the rigid Hessian matrices (S1, S2, S3) were done using Fogarasi's and Pulay's fctint code, ${ }^{8}$ while the relaxed force constants (S4) were computed using our COMPLIANC 3.0 code, freely available from our site http://www.oc.tubs.de/Grunenberg (Fig. 1).

As expected (see Table 1), our relaxed diagonal boron-boron

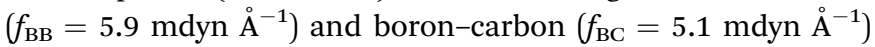
force constants comprise a lower boundary for all other possible coordinate systems. While the effect is quite small for Schnöckel's (S1) and Pulay's (S2) coordinate systems - both $f_{\mathrm{BB}}$ values are 6.0 mdyn $\AA^{-1}$ - the $f_{\mathrm{BB}}$ value expressed in $z$-matrix variables (S3) of 7.3 mdyn $\AA^{-1}$ again is "pretending" a stronger BB bond. The same is true for the boron-carbon bond. ${ }^{9}$ Most important nevertheless, the true coupling between the boronboron bond and the boron-carbon bond, after the elimination

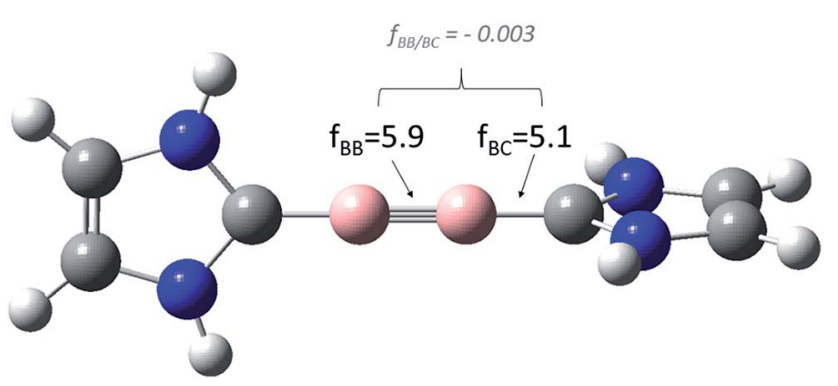

Fig. 1 Relevant relaxed force and coupling constants, computed at the BP86/dz level of theory, applying the generalized compliance constants (GCC) approach, ${ }^{6}$ for the model system 1 . Both, the diagonal and the coupling terms are unique, that means, they do not depend on the definition of all other coordinates. 
Table 1 Relevant relaxed force and coupling constants in mdyn $\AA^{-1}$ (right column), computed at the BP86/dz level of theory, applying the generalized compliance constants (GCC) approach by Brandhorst and Grunenberg, ${ }^{6}$ as well as three different rigid force constants coordinate systems for the model system 1

\begin{tabular}{lllll}
\hline & $(\mathrm{S} 1)$ & $(\mathrm{S} 2)$ & $(\mathrm{S} 3)$ & $(\mathrm{S} 4)$ \\
\hline$f_{\mathrm{BB}}$ & 6.0 & 6.0 & 7.3 & $\mathbf{5 . 9}$ \\
$f_{\mathrm{BC}}$ & 5.2 & 5.2 & 5.4 & $\mathbf{5 . 1}$ \\
$f_{\mathrm{BB} / \mathrm{BC}}$ & 0.16 & 0.11 & 0.38 & $\mathbf{- 0 . 0 0 3}$
\end{tabular}

${ }^{a}$ The unit of the coupling constant is given in $\AA \mathrm{mdyn}^{-1}$.

of all numerical artifacts, is more or less negligible $\left(f_{\mathrm{BB} / \mathrm{BC}}=\right.$ $-0.003)$.

In combination with a quick analysis of three model systems of archetypical B-B single, double and triple bonds and a comparison with the relaxed force constant of 6.3 mdyn $\AA^{-1}$ computed by Fischer and Braunschweig ${ }^{10}$ for the real diboryne $\left(\mathrm{B}_{2} \mathrm{IDip}_{2}\right)$ allows a unique interpretation of the $\mathrm{B}-\mathrm{B}$ bond under question as a triple bond. ${ }^{\mathbf{1 1}}$

$\begin{array}{lll}\mathrm{NHC}_{2} \mathrm{H}_{2} \mathrm{BBH}_{2}-\mathrm{NHC} & \text { (B-B single bond): } & 1.5 \mathrm{mdyn} \mathrm{A}^{-1} \\ \mathrm{NHC}-\mathrm{HBBH}-\mathrm{NHC} & \text { (B=B double bond): } & 3.8 \mathrm{mdyn} \mathrm{A}^{-1} \\ \mathrm{NHC}-\mathrm{BB}-\mathrm{NHC} & \text { (B=B triple bond): } & 6.5 \mathrm{mdyn} \mathrm{A}^{-1}\end{array}$

\section{Conclusions}

(1) Rigid coupling force constants, as applied by Schnöckel and Köppe, are ill-defined and hence invalid as bond strength descriptors. The numerical values depend on the definition of all other coordinates. Relaxed force constants, on the other hand, do not depend on the coordinate system. They address the question "which force has to be applied against a specific internal coordinate in order to achieve a given displacement, while all other forces thereby introduced are allowed to relax". The displacements of all other coordinates caused by these forces are given by the compliance coupling constants, which are the off-diagonal terms of the compliance matrix.

(2) If rigid force constants are employed for the description of individual bond strength anyhow, the bonding situation is always described as being too strong, since the values of rigid force constants are necessarily higher than the values of the corresponding relaxed force constants. This is of course also true for non-covalent interactions, ${ }^{12}$ even if there are still some misunderstandings on this aspect. ${ }^{13}$

(3) The true coupling between the boron-boron bond and the boron-carbon bond, after the elimination of all numerical artifacts, is negligible $\left(f_{\mathrm{BB} / \mathrm{BC}}=-0.003\right)$.

Applying the method of generalized compliance constants $(G C C)$, the calculation of relaxed force constants for covalent and non-covalent coordinates is now a straightforward task. It is somehow disturbing that, nearly 15 years after our original publication, ${ }^{\mathbf{1 4}}$ the use of ill-defined, rigid force constants as bond strength descriptors is still prevalent.

\section{References}

1 (a) J. Grunenberg, Nat. Chem., 2012, 4, 154-155; (b) K. Brandhorst, J. Grunenberg and M. Tamm, J. Chem. Educ., 2008, 85, 1692-1695.

2 R. Köppe and H. Schnöckel, Chem. Sci., 2015, 6, 1199-1205.

3 H. Braunschweig, R. D. Dewhurst, K. Hammond, J. Mies, K. Radacki and A. Vargas, Science, 2012, 336, 1420.

4 In this context it is important to highlight the matrix properties of force constants, that is, the existence of both, diagonal and off-diagonal elements: J. Grunenberg, Angew. Chem., Int. Ed., 2001, 40, 4027-4029.

5 J. Grunenberg, J. Am. Chem. Soc., 2004, 126, 16310-16311. 6 (a) G. von Frantzius, R. Streubel, K. Brandhorst and J. Grunenberg, Organometallics, 2006, 25, 118; (b) K. Brandhorst and J. Grunenberg, J. Chem. Phys., 2010, 132, 184101-184107.

7 (a) K. Brandhorst and J. Grunenberg, ChemPhysChem, 2007, 8, 1151-1156; (b) K. Brandhorst and J. Grunenberg, Chem. Soc. Rev., 2008, 37, 1558-1567; (c) G. Markopoulos and J. Grunenberg, Angew. Chem., Int. Ed., 2013, 52, 10648-10651. 8 G. Fogarasi, X. Zhou, P. W. Taylor and P. Pulay, J. Am. Chem. Soc., 1992, 114, 8191-8201.

9 Our calculated relaxed force constants for a typical $\mathrm{C}=\mathrm{B}$ double and $\mathrm{C} \equiv \mathrm{B}$ triple bond are 8.93 (in $\mathrm{Me}-\mathrm{B}=\mathrm{CMe}_{2}$ ) and 11.11 mdyn $\AA^{-1}$ in $[\mathrm{Me}-\mathrm{C} \equiv \mathrm{B}-\mathrm{Me}]^{-}$, respectively. The values for a typical $\mathrm{CC}$, single, double and triple bond are 4.0, 9.5 and 17.3 mdyn $\AA^{-1}$, respectively, see ref. 4 .

$10 \mathrm{~J}$. Böhnke, H. Braunschweig, P. Constantinidis, T. Dellermann, W. C. Ewing, I. Fischer, K. Hammond, F. Hupp, J. Mies, H.-Ch. Schmitt and A. Vargas, J. Am. Chem. Soc., 2015, 137, 1766-1769.

11 For a recent publication analyzing three different B-B multiple bond descriptors (AIM, NBO and compliance constants) see: M. D. Walter, et al., Eur. J. Inorg. Chem., 2013, 4097-4104.

12 (a) S. S. Zhu, H. Staats, K. Brandhorst, J. Grunenberg, F. Gruppi, E. Dalcanale, A. Lützen, K. Rissanen and C. A. Schalley, Angew. Chem., Int. Ed., 2008, 47, 788-792; (b) A. G. Ponomareva, Y. P. Yurenko, R. O. Zhurakivsky, T. van Mourik and D. M. Hovorun, Phys. Chem. Chem. Phys., 2012, 14, 6787-6795; (c) J. Grunenberg and G. Barone, RSC Adv., 2013, 3, 4757-4762.

13 See, for example: J. Baker, Wiley Interdiscip. Rev.: Comput. Mol. Sci., 2014, 4, 111-115.

14 J. Grunenberg and N. Goldberg, J. Am. Chem. Soc., 2000, 122, 6045-6047. 